Peripheral Nerve Injuries: A Clinical Guide 

Rolfe Birch

\section{Peripheral Nerve Injuries: A Clinical Guide}

祭 Springer 
Rolfe Birch MChir. FRCP\&S (Glas) FRCS

(Edin) FRCS Eng (by election)

Professor in Neurological Orthopaedic Surgery

University College

London

UK

\author{
Visiting Professor \\ Department of Academic Neurology \\ Imperial College \\ London \\ UK
}

\author{
Late Head of Department \\ Peripheral Nerve Injury Unit \\ Royal National Orthopaedic Hospital \\ London \\ UK
}

Honorary Orthopaedic Surgeon to the Royal

Navy, UK

Consultant in Charge

War Nerve Injury Clinic at the Defence

Medical Rehabilitation Centre

Headley Court, Leatherhead, Surrey

UK

ISBN 978-1-4471-4612-4

ISBN 978-1-4471-4613-1 (eBook)

DOI 10.1007/978-1-4471-4613-1

Springer London Heidelberg New York Dordrecht

Library of Congress Control Number: 2012952506

(C) Springer-Verlag London 2013

This work is subject to copyright. All rights are reserved by the Publisher, whether the whole or part of the material is concerned, specifically the rights of translation, reprinting, reuse of illustrations, recitation, broadcasting, reproduction on microfilms or in any other physical way, and transmission or information storage and retrieval, electronic adaptation, computer software, or by similar or dissimilar methodology now known or hereafter developed. Exempted from this legal reservation are brief excerpts in connection with reviews or scholarly analysis or material supplied specifically for the purpose of being entered and executed on a computer system, for exclusive use by the purchaser of the work. Duplication of this publication or parts thereof is permitted only under the provisions of the Copyright Law of the Publisher's location, in its current version, and permission for use must always be obtained from Springer. Permissions for use may be obtained through RightsLink at the Copyright Clearance Center. Violations are liable to prosecution under the respective Copyright Law.

The use of general descriptive names, registered names, trademarks, service marks, etc. in this publication does not imply, even in the absence of a specific statement, that such names are exempt from the relevant protective laws and regulations and therefore free for general use.

While the advice and information in this book are believed to be true and accurate at the date of publication, neither the authors nor the editors nor the publisher can accept any legal responsibility for any errors or omissions that may be made. The publisher makes no warranty, express or implied, with respect to the material contained herein.

Printed on acid-free paper

Springer is part of Springer Science+Business Media (www.springer.com) 


\section{Contents}

1 The Peripheral Nervous System: Anatomy and Function ................. 1

1.1 The Cranial Nerves ....................................................................... 4

1.2 The Spinal Nerves .................................................................... 5

1.2.1 The Anterior Primary Rami ................................................ 5

1.2.2 Thoracic Anterior Primary Rami ........................................ 9

1.2.3 Lumbar and Sacral Anterior Primary Rami...................... 10

1.2.4 The Posterior Primary Rami .............................................. 18

1.3 The Autonomic Nervous System.................................................. 20

1.3.1 The Sympathetic System ................................................. 21

1.3.2 The Parasympathetic Nervous System .............................. 23

1.4 Nerves at Risk from Musculo Skeletal Injury .............................. 25

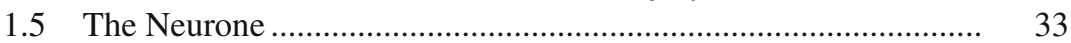

1.5.1 The Neurotrophins ........................................................ 34

1.6 The Nerve Fibre..................................................................... 38

1.6.1 The Axon .................................................................... 40

1.6.2 Axonal Transport ........................................................... 40

1.6.3 The Glial Cells of the Peripheral Nervous System ........... 42

1.6.4 The Myelin Sheath.............................................................. 43

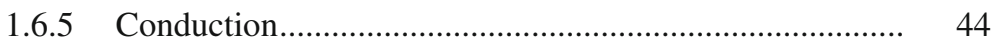

1.6.6 The Basis of the Action Potential: Ion Channels............. 46

1.7 The Peripheral Nerve Trunk ............................................................ 47

1.7.1 The Connective Tissue Sheaths .......................................... 48

1.7.2 Topographical Organisation.............................................. 48

1.7.3 The Blood Supply of Nerves ............................................. 50

1.7.4 The Nervi Nervorum......................................................... 51

1.8 Changes in Nerves with Aging .................................................. 51

1.9 The Somatic Motor System ............................................................. 51

1.10 The Somatic Sensory System ....................................................... 53

1.10.1 Cutaneous Sensibility ……………………………….... 54

1.10.2 The Skin ...................................................................... 55 
1.10.3 Cutaneous Sensory Receptors.......................................... 56

1.10.4 Deep Sensibility ............................................................ 58

1.10.5 Central Connections............................................................ 60

1.10.6 Afferent Autonomic Pathways......................................... 62

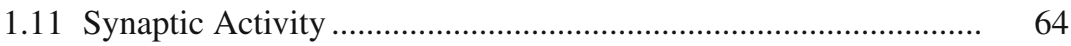

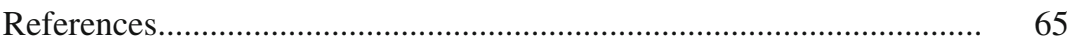

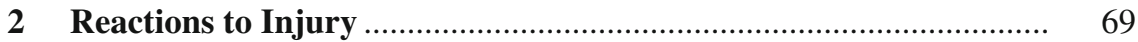

2.1 Conduction Block: Neurapraxia ................................................... $\quad 72$

2.1.1 Transient Ischaemia ......................................................... 72

2.1.2 Conduction Block in Myelin Deformation and Focal Demyelination ............................................. 73

2.1.3 The Conduction Block of War Wounds ............................ 74

2.2 The Degenerative Lesion .............................................................. 75

2.2.1 The Cell Body and Proximal Stump ................................... 75

2.2.2 The Distal Stump ………………………………........... 78

2.2.3 Contralateral Effects ....................................................... 79

2.3 The Special Case of the Brachial Plexus ....................................... $\quad 82$

2.4 Types of Lesion Produced by Different Physical Agents .............. $\quad 84$

2.4.1 Acute Ischaemia............................................................... 85

2.4.2 Ischaemia from Tamponade.............................................. 86

2.4.3 Ischaemia and Acute Compression Within Neurovascular Fascial Compartments ............................ $\quad 89$

2.4.4 Ischaemia by Acute Compression from Swollen Muscle..................................................................... 90

2.4.5 Ischaemia Caused by Traction ............................................. 93

2.4.6 Compression: Acute........................................................ 95

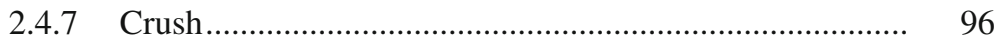

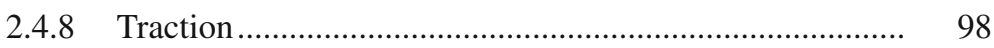

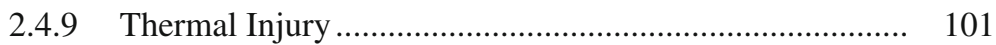

2.4.10 Electric Shock ............................................................. 102

2.4.11 Injection Injury ........................................................... 105

2.4.12 Vibration Injury ............................................................... 107

2.4.13 Radiation and Peripheral Nerves .................................. 107

References............................................................................. 109

$3 \quad$ Regeneration and Recovery of Function ......................................... 113

3.1 The Response of the Nerve and Axon to Transection .................. 114

3.1.1 The Schwann Cells ........................................................ 115

3.1.2 The Axon ................................................................... 115

3.1.3 Reorganisation into "Mini Fascicles" ............................ 116

3.1.4 Guidance and Selection .................................................. 116

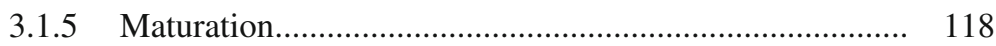

3.1.6 The Rate of Regeneration ............................................... 118 
3.2 Regeneration of End-Organs ................................................... 119

3.2.1 The Cutaneous Sensory Receptors ................................. 119

3.3 Regeneration After Intradural Injury ....................................... 120

3.4 The Repair of Large Gaps ....................................................... 122

3.5 Complications of Regeneration ........................................... 123

3.5.1 Muscle Function ........................................................ 123

3.5.2 Pain .................................................................... 124

3.6 Recovery of Function After Nerve Repair............................... 126

3.6.1 Duration of Study: Records ........................................ 127

3.6.2 Grading of Results ................................................... 128

3.7 Factors Governing Prognosis................................................... 136

3.7.1 Severity of Injury .................................................... 136

3.7.2 Delay ....................................................................... 137

3.7.3 Other Factors in Prognosis.......................................... 138

3.8 Conclusion ...................................................................... 140

References....................................................................... 140

4 Clinical Aspects of Nerve Injury .................................................... 143

4.1 The History: Characteristics of the Wound ............................... 143

4.2 Associated Symptoms and Signs ............................................ 146

4.2.1 Examination .............................................................. 147

4.3 Recognition of the Level and the Depth of Injury ..................... 149

4.3.1 Level ........................................................................ 149

4.3.2 Depth............................................................... 152

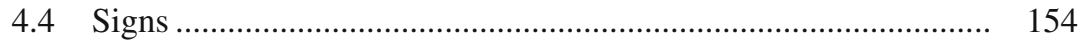

4.5 Tinel's Sign ........................................................................ 157

4.5.1 Eliciting the Tinel Sign in Closed Lesions .................... 160

4.5.2 Tinel's Sign and Recovery ......................................... 162

4.6 Examination of Sensibility .................................................... 163

4.6.1 Quantitative Sensory Testing (QST) ........................... 167

4.7 Examination of Muscles ......................................................... 168

4.7.1 Some Pitfalls .............................................................. 168

4.7.2 Measurement of Muscle Power ..................................... 169

4.8 Some Difficulties in Diagnosis ............................................. 171

4.8.1 Thoraco Scapular, Thoraco Humeral, and Scapulo Humeral Muscles ................................................... 172

4.8.2 The Hand ...................................................................... 178

4.8.3 The Lower Limb .......................................................... 179

4.9 Late Signs of Nerve Injury .................................................... 180

4.10 The Diagnosis of Neuropathic Pain After Injury to a Nerve........ 184

4.10 .1 Allodynia ................................................................. 185

4.10.2 Hyperpathia........................................................... 187

4.10.3 Deafferentation Pain ................................................. 187 
4.10.4 Clinical Assessment.................................................. 188

4.10.5 Neuropathic Pain Syndromes......................................... 189

4.11 Aids to Diagnosis ................................................................. 191

4.11.1 Neurophysiological Investigations (NPI) ...................... 191

4.11.2 High Resolution Ultrasonography ............................... 192

References........................................................................... 193

5 Operating on Peripheral Nerves....................................................... 195

5.1 Indications and Objects of Intervention.................................... 195

5.1.1 The Lessons of War ..................................................... 195

5.1.2 Timing: Nerve Lesions in Fractures and Dislocations..................................................... 196

5.2 General Principles of Operation ............................................... 198

5.2.1 Control of Bleeding ................................................... 198

5.2.2 Preparation ................................................................. 199

5.2.3 Prevention of Pain......................................................... 200

5.2.4 Apparatus and Instruments ......................................... 200

5.2.5 Incisions: Handling of Tissues...................................... 204

5.2.6 The Record............................................................... 206

5.3 Methods of Repair ................................................................. 207

5.3.1 The Vascular Repair...................................................... 207

5.3.2 The Nerve Operations .................................................. 209

5.3.3 Biopsy ...................................................................... 211

5.4 The Nerve Repair................................................................... 214

5.4.1 Methods of Suture...................................................... 217

5.4.2 Grafting..................................................................... 219

5.4.3 Indications for and Methods of Nerve Transfer.............. 223

5.4.4 Other, Non Neural, Material for Grafts: Entubation........ 229

5.4.5 Immobilisation........................................................ 231

5.5 Approaches to Individual Nerves: Neck and Upper Limb ........... 234

5.5.1 The Transverse Supraclavicular Approach:

(Anterior, or Anterolateral) ...................................... 234

5.5.2 The Transclavicular Exposure ...................................... 239

5.5.3 The Postero-Lateral Route .............................................. 241

5.5.4 The Spinal Accessory Nerve.......................................... 244

5.5.5 The Suprascapular Nerve............................................. 245

5.5.6 The Infraclavicular Part of the Brachial Plexus.............. 245

5.5.7 The Circumflex Nerve .............................................. 247

5.5.8 Median and Ulnar Nerves in the Arm and the Axilla...... 247

5.5.9 The Radial Nerve ......................................................... 248

5.5.10 The Posterior Interosseous Nerve .................................... 250

5.5.11 The Lower Part of the Median Nerve ............................. 251

5.5.12 The Lower Part of the Ulnar Nerve ............................... 254 
5.6 Approaches to Individual Nerves: Abdomen, Pelvis and Lower Limb 255

5.6.1 The Lumbar Plexus ....................................................... 255

5.6.2 The Femoral Nerve ....................................................... 256

5.6.3 The Sciatic Nerve......................................................... 257

5.6.4 The Tibial and Common Peroneal Nerves in the Popliteal Fossa and Below .................................. 260

5.6.5 The Lower Tibial Nerve and the Plantar Nerves ............. 260

References.................................................................................... 263

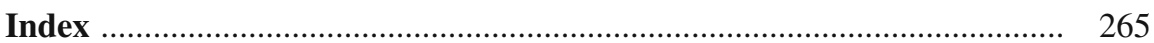


